\title{
Energetic Oxygen and Sulfur in the Jovian Magnetosphere
}

\author{
N. Gehrels AND E. C. Stone \\ California Institute of Technology, Pasadena, California 91125
}

J. H. TRAINOR

Goddard Space Flight Center, Greenbelt, Maryland 20771

\begin{abstract}
This paper reports measurements made by the cosmic ray subsystem onboard Voyager 1 and 2 in the Jovian magnetosphere. Energy spectra of oxygen ions in the energy range 1-20 MeV/nuc between 5 and $20 R_{J}$ are presented and used to calculate phase space densities. There is a steep positive radial gradient in the phase space density of the energetic oxygen ions in this region, indicating an inward diffusive flow. Solutions of the diffusion equation assuming a diffusion coefficient $D$ and loss lifetime $\tau$ of the forms $D=$ $D_{0} L^{n}$ and $\tau=\tau_{0} L^{m}$, where $D_{0}, \tau_{0}, n$, and $m$ are constants, and $L$ is the Mcllwain parameter, are fit to the radial phase space density profile of oxygen ions with magnetic moments of $680 \mathrm{MeV} / \mathrm{nuc}-\mathrm{G}$. The best fits are found to have $n+m \approx 6$ and $3<n<6$. On the basis of the diffusion coefficient upper limit obtained from these fits, the upper limit on the rate at which oxygen ions with $>400 \mathrm{MeV} / \mathrm{nuc}-\mathrm{G}$ diffuse inward across $10 R_{J}$ is $5 \times 10^{21 \pm 1}$ ions $\mathrm{s}^{-1}$. The observations suggest that oxygen and sulfur ions in the Io plasma torus diffuse radially outward, are nonadiabatically accelerated in some region outside $17 R_{J}$ and then diffuse inward and outward from the acceleration region.
\end{abstract}

\section{INTRODUCTION}

Observations made in the Jovian magnetosphere of nuclei with charge greater than 2 and energies between 7 and 14 MeV per nucleon (MeV/nuc) [Vogt et al., 1979a, b] have shown that, beyond $\sim 25$ Jovian radii $\left(R_{J}\right)$, the elemental composition is similar to that of the solar energetic particles. In the inner magnetosphere ( $\leqslant 12 R_{J}$ ), however, the composition is drastically different, dominated by oxygen and sulfur with sodium also present. Observations between 0.60 and $1.15 \mathrm{MeV} /$ nuc [Hamilton et al., 1981] show a similar sharp increase in the oxygen and sulfur abundances relative to helium and carbon inside $\sim 20 R_{\boldsymbol{J}}$.

The likely source of the oxygen, sodium, and sulfur is the Jovian satellite Io. Gaseous $\mathrm{SO}_{2}$ is a major constituent of Io's atmosphere [Pearl et al., 1979; Kumar, 1979] and is probably the volatile that drives Io's volcanism [Smith et al., 1979; Johnson et al., 1979]. Oxygen and sulfur are also the dominant species at plasma energies in the lo torus [Broadfoot et al., 1979; Sandel et al., 1979; Bagenal and Sullivan, 1981]. The evidence that sodium is associated with Io comes from earth-based observations of neutral sodium optical emission [Brown, 1974; Brown and Yung, 1976].

Since the ions in the plasma near Io typically have eV/nuc energies [Bagenal and Sullivan, 1981], an acceleration process is required to produce the $\mathrm{MeV} / \mathrm{nuc}$ ions observed. Measurements of the gradient in the ion density can be used to calculate diffusive flow directions and can thereby indicate the location of the acceleration site. A preliminary analysis using Voyager 2 data between 10 and $13 R_{J}$ [Vogt et $a l$., 1979b] determined that there is a positive radial gradient in the phase space density of the high-energy oxygen ions, indicating an inward diffusive flow. This observation suggests that the acceleration region is outside $13 R_{J}$. Only oxygen was analyzed due to statistical considerations.

In the present analysis this earlier work is extended to include both Voyager 1 and 2 data between 5 and $17 R_{J}$. The observations are compared with various radial diffusion models and are used to derive limits on the acceleration efficiency.

Copyright (C) 1981 by the American Geophysical Union.

\section{PARTICle FluXes AND ENERgY SPECTRA}

Data from the cosmic ray subsystem (CRS) low-energy telescopes (LETs) [Stone et al., 1977; Stilwell et al., 1979] were used in this analysis. A schematic diagram of a LET is shown in Figure 1. The LET system consists of four such telescopes, LET A, B, C, and D, oriented to allow anisotropy measurements. LET $A$ and $C$ were turned off inside $\sim 20 R$, on both Voyagers to reduce the noise level at the input of the pulseheight analyzers shared with LET B and D, respectively. Also, the LET $B$ and $D L 4$ detectors, normally used in active anticoincidence against penetrating particles, were turned off inside $\sim 20 R_{J}$ to minimize accidental event rejection in the high-radiation environment of the inner magnetosphere. The orientation of the instrument in this region on both Voyagers was such that LET $B$ pointed approximately perpendicular to the local magnetic field direction when the spacecraft was near the magnetic equatorial plane, while LET $D$ was more nearly field aligned. To simplify the analysis that follows, consideration will be restricted to particles with pitch angles of $\sim 90^{\circ}$ to the field, and thus only LET $B$ will be used. A summary of the proton measurements made by the LET system in the Jovian magnetosphere is given by Schardt et al. [1981].

The most important feature of the LET for the present study is that it provides multiparameter analysis of individual nuclei with $\mathrm{MeV} /$ nuc energies and nuclear charge $\boldsymbol{Z}$, greater than 2, with an rms charge resolution of $0.1<\sigma_{z}<0.5$ charge units, where the larger values occur during high flux periods. Since incoming fast ions are stripped of their electrons in the 3- $\mu \mathrm{m} \mathrm{Al}$ window of the telescope, no information is available about the charge state of the ions prior to entry. The LET system also provides measurements of the counting rate of particles with energy losses $E 1, E 2$, and $E 3$ in detectors $L 1, L 2$, and $L 3$ such that $E 1+0.4 E 2+0.2 E 3>9.6 \mathrm{MeV}$. Because the geometry factor defined by the collimator and $L 1\left(4.6 \mathrm{~cm}^{2} \mathrm{sr}\right)$ is much larger than that defined by $L 1$ and $L 2\left(0.44 \mathrm{~cm}^{2} \mathrm{sr}\right)$, this rate is primarily that of particles losing more than $9.6 \mathrm{MeV}$ in $L 1$. It is called the $Z>2$ rate since neither protons nor alpha particles within the acceptance cone of the telescope can lose as much as $9.6 \mathrm{MeV}$ in a $35-\mu \mathrm{m}$ silicon detector. Table 1 lists 


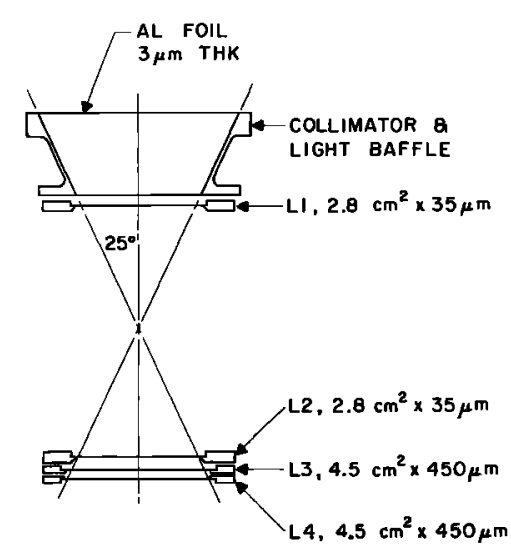

LOW ENERGY TELESCOPE (LET)

Fig. 1. Cross-section of a low-energy telescope (LET). All detectors are sillicon surface-barrier detectors.

the geometry factors and the oxygen and sulfur energy ranges for the $Z>2$ rate and for event analysis.

Plots of the livetime-corrected $Z>2$ flux as a function of time for the Voyager 1 and 2 encounters are shown in Figures $2 a$ and $2 b$, respectively. The livetime corrections are based on the calibration of a spare CRS instrument (see the appendix). Inside $6.5 R_{J}$, the $Z>2$ flux exhibits minima at $L=5.6$ inbound (day 64, 0934:24 \pm 0001:36 UT) and 5.4 outbound (day 64, 1406:24 \pm 0001:36 UT), where the Mcllwain parameter $L$ is taken to be the zenocentric distance of the spacecraft in units of $R_{J}$ at these minima. The difference between these $L$ values and the actual $L$ values at the minima, owing to the spacecraft dipole latitude $\left(\sim 5^{\circ}\right)$ and the dipole offset, is less than $\sim 0.1$. The locations of the observed minima indicate that the particle losses from the observed energy range are caused by the Io plasma torus rather than Io itself since measurements of the plasma in the torus showed sharp decreases in plasma density just inside the peak densities at $L=5.7$ inbound (peak \#2 at day 64, 0924 UT) and 5.6 outbound (day 64, 1420 UT) [Bagenal and Sullivan, 1981], whereas Io is never inside $L=5.8$. Also, it was observed on the Voyager 1 inbound pass that the electromagnetic wave activity associated with the torus stopped abruptly at $\sim 5.6 R$, [Scarf et al., 1979]. This abrupt decrease of the wave activity inside $\sim 5.6 R_{J}$ may be the direct cause of the inbound particle flux minimum since the particle losses are likely due to wave-particle interactions. Note that minima in flux measurements above a fixed energy threshold do not necessarily correspond to minima in the phase space densities of the particles. As will be shown, the flux minima near Io correspond to a change in the radial gradient rather than a minimum in the phase space density profile. The two decreases at $\sim 9.4 R$, inbound and outbound were caused by Europa. In contrast, the large scale structure in the Voyager 2 plot is due to varying distance of the spacecraft from the magnetic equatorial plane. The peaks at day 190, $1400 \mathrm{UT}, 2200-2400 \mathrm{UT}$, and day 191, $0900 \mathrm{UT}$ are at times when the spacecraft crossed this plane. The fine scale structure in this flux is not yet understood. The inbound $Z>2$ flux is compared for the two spacecraft as a function of radial distance from Jupiter in Figure 3. Inside $18 R_{J}$, the Voyager 1 and 2 fluxes were the same to within a factor of $\sim 2$. There is therefore no evidence for major temporal variations in the heavy ion fluxes in this region between the two encounters, and in the analysis that follows, we will consider both sets of measurements to be of the same stable population.

Oxygen energy spectra have been calculated for the six periods indicated in Figure 2 and listed in Table 2. Periods 2 and 3 for Voyager 1 are each the combination of inbound and outbound time intervals ( $a$ and $b$ ) symmetrically spaced with respect to the flux minima near Io. These six periods are the only ones in the data set inside $18 R_{J}$ for which there are a statistically significant number of pulse-height analyzed events, and for which LET $B$ was pointing within $20^{\circ}$ of the plane perpendicular to the local $B$ field direction. This last criterion simplifies the phase space density analysis that follows.

A plot of all three-detector events $(L 1 \cdot L 2 \cdot L 3)$ which were pulse-height analyzed by LET $B$ in regions 1,2 , and 3 is shown in Figure 4. The two tracks on the plot are due to oxygen and sulfur events that penetrated $L 1$ and $L 2$ and stopped in $L 3$. The events along the left edge of the plot are predominantly oxygen and sulfur events that penetrated $L 1$ and stopped in $L 2$ and, in addition, had a small pulse in $L 3$, most likely due to an accidentally coincident proton (or several electrons). These events are identified by the tracks they produced on an $E 1$ versus $E 2$ plot. In general, different livetime corrections were required for the different types of events, as discussed in the appendix.

The differential energy spectra of the oxygen events in regions 2 and 4 are shown in Figure 5. A striking feature in the region 2 spectrum is the peak at $\sim 5.5 \mathrm{MeV} /$ nuc. In order to extend these spectra to lower energies, we convert to integral spectra and add a point at $\sim 1.2 \mathrm{MeV} /$ nuc (energy of particle incident on the telescope window) calculated from the $Z>2$ rate. Since the $Z>2$ rate is made up of both oxygen and sulfur (and traces of other elements) in the inner magnetosphere, one must measure or estimate the sulfur to oxygen ratio in order to obtain an integral flux of either species alone. In regions 1, 2, and 3, a pulse-height histogram from the $L 1$ detector reveals two distinct maxima as shown in Figure 6. The maximum at $\sim 30 \mathrm{MeV}$ can be understood as the energy loss of 5-6 MeV/nuc oxygen ions penetrating the detector. This identification is reasonable since the differential oxygen spectrum is peaked at $\sim 6 \mathrm{MeV} /$ nuc. Similarly, a differential sulfur spectrum peaked at $\sim 5 \mathrm{MeV} /$ nuc would produce the feature at $\sim 10 \mathrm{MeV}$. Therefore, in these regions, the sulfur to oxygen

TABLE 1. Nominal LET Parameters

\begin{tabular}{cccc}
\hline & Geometry Factor, ${ }^{*} \mathrm{~cm}^{2} \mathrm{sr}$ & Element & Energy Range, $\dagger$ Mev/nuc \\
\hline$Z>2$ rate & 4.6 & Oxygen & $1.1-\sim 25$. \\
Event analysis & \multirow{2}{*}{0.44} & Sulfur & $0.9-\sim 120$. \\
$(L 1 \cdot L 2)$ & & Oxygen & $3.6-\sim 23$. \\
\hline
\end{tabular}

* Each of four telescopes.

$\dagger$ Energy loss in the 3- $\mu \mathrm{m} \mathrm{Al}$ window (see Figure 1) has been added to the detector threshold to give the incident energy of the particle. 

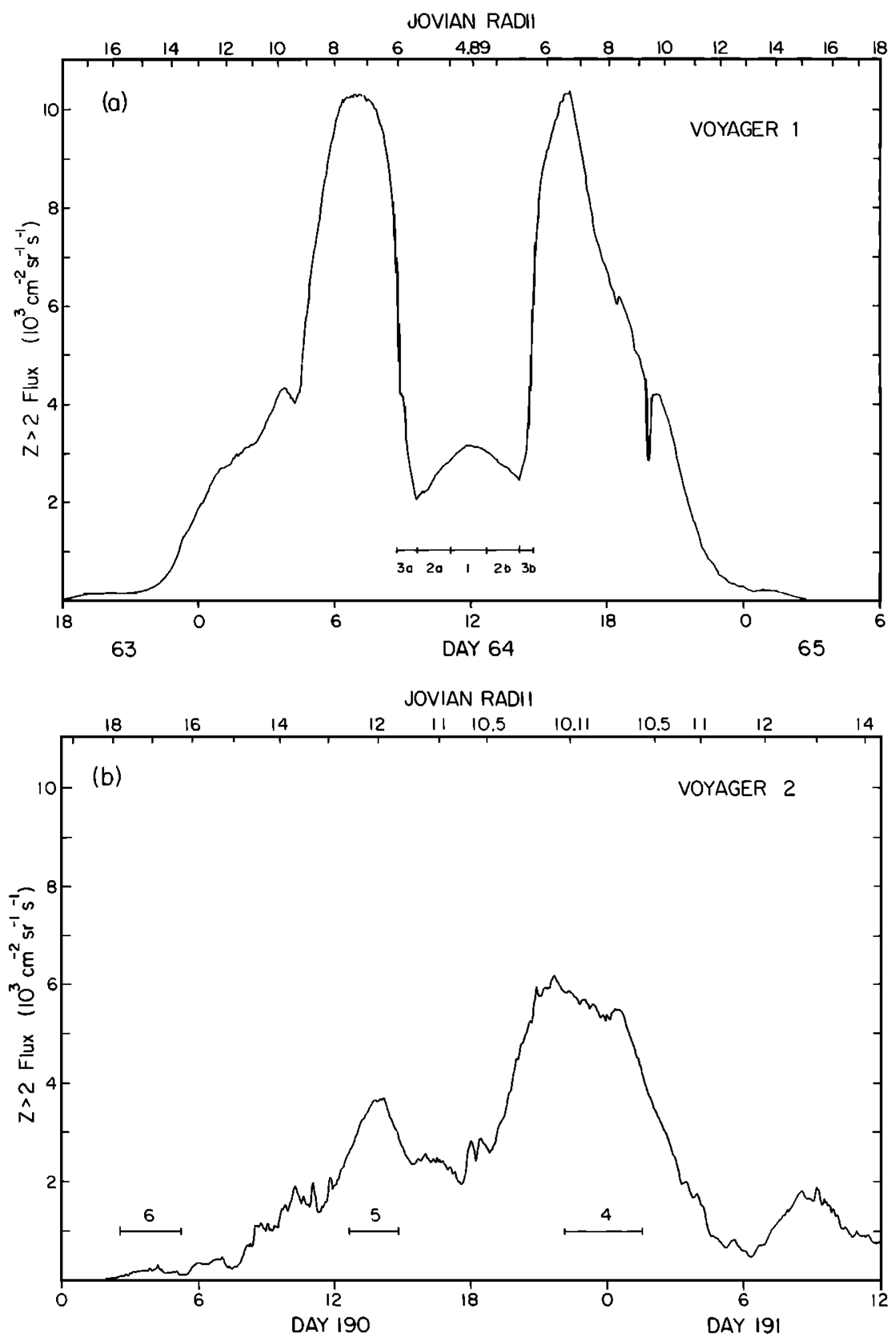

Fig. 2. Livetime-corrected $Z>2$ flux (see Table 1) for (a) Voyager 1 and $(b)$ Voyager 2 closest approach. The day number means day of year 1979 . The numbered time intervals refer to regions in which oxygen spectra have been calculated (Table 2). Note that the vertical scale is linear.

ratio in the $Z>2$ rate can be directly determined. In regions 4,5 , and 6 , the $L 1$ pulse height histograms do not have separate oxygen and sulfur maxima, so the ratio cannot be measured directly. In these regions the ratio was estimated using $L 1 \cdot L 2$ data. Table 3 lists the values of the oxygen fraction of the $Z>2$ rate used for the different regions. Note that, since the oxygen and sulfur energy thresholds for this rate are different, the numbers are not equal energy-per-nucleon abundance fractions. The exact oxygen threshold energy for the $Z$ $>2$ rate must also be determined in order to use the $Z>2$ flux as a point in the integral spectrum. The threshold level varies between 1.1 and $1.4 \mathrm{MeV} /$ nuc due to discriminator threshold shifts caused by high flux levels, and was measured during the calibration of the spare CRS instrument (see the appendix). The results are listed for each region in Table 3.
The oxygen integral energy spectra in regions 2 and 4, including the $Z>2$ points, are shown in Figure 7. The flattening of the spectrum in region 2 below $\sim 5.5 \mathrm{MeV} /$ nuc corresponds to the decrease in differential intensity seen in Figure 5. The spectrum remains flat between 3.5 and 1.4 $\mathrm{MeV} / \mathrm{nuc}$, which implies that in this interval the differential intensity remains below the peak value of $\sim 300\left(\mathrm{~cm}^{2} \mathrm{sr} s\right.$ $\mathrm{MeV} / \mathrm{nuc})^{-1}$. A likely cause of this flattening in the integral spectrum is that particle loss mechanisms near Io are more efficient for lower energy particles. Pioneer 10 observations showed such a preferential loss of lower energy protons near Io between 1 and $20 \mathrm{MeV}$ [Trainor et al., 1974]. To use the two spectra presented in this section, as well as spectra from other regions, for gradient and flow direction determinations, and thus restrict the region where the energetic ions are acceler- 


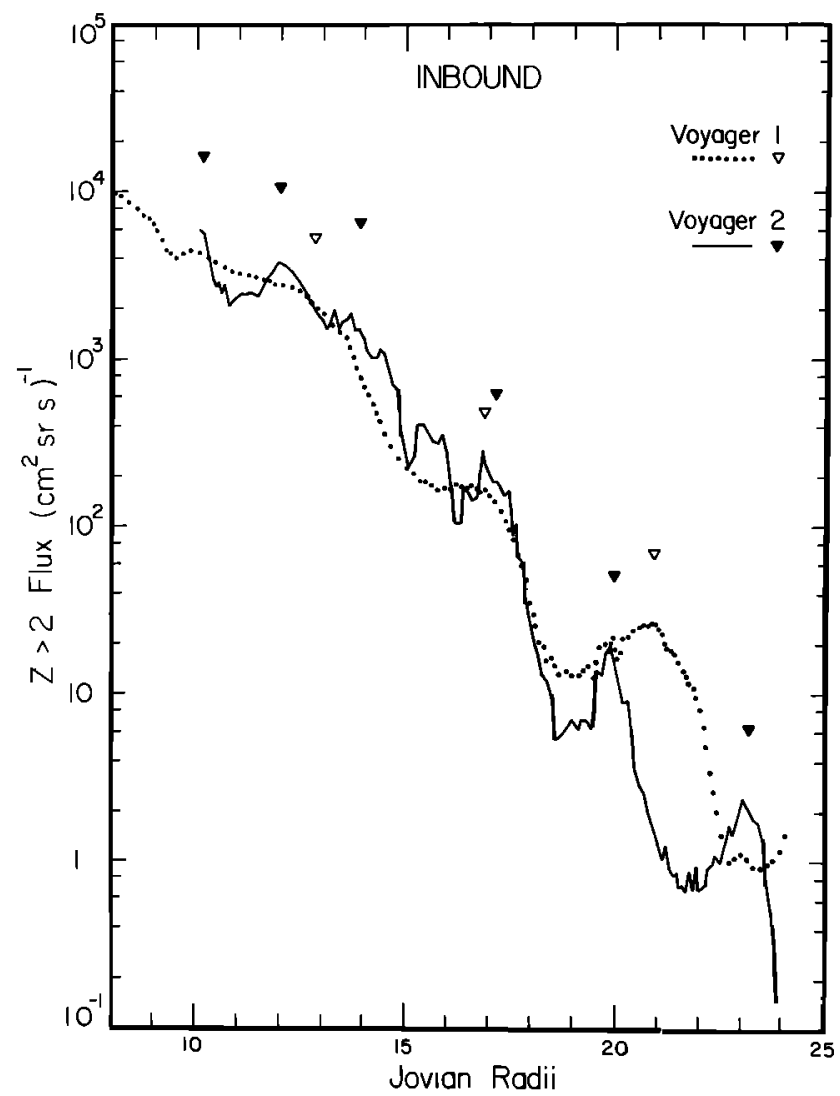

Fig. 3. Voyager 1 and 2 livetime corrected $Z>2$ fluxes as functions of spacecraft distance from Jupiter, inbound. The triangles indicate magnetic equatorial crossings.

ated, it is useful to relate the intensity measurements to a diffusion theory.

\section{COMPARISON OF SPECTRA AND GRADIENT DETERMINATION}

The radial diffusion theory that will be adopted for the present analysis assumes that particles conserve their first and second adiabatic invariants but not their third in the diffusion process [see e.g., Schulz and Lanzerotti, 1974]. Conservation of the first invariant or magnetic moment, $M=p_{1}{ }^{2} / 2 m B$ where $m$ is the particle mass, $B$ is the magnetic field strength, and $p_{\perp}$ is the momentum perpendicular to the $B$ field direction, gives a linear scaling of energy with magnetic field strength, $E=$ $M B$ (nonrelativistic), for the special case of mirroring particles (pitch angle, $\alpha=90^{\circ}$ ). For these particles, $p=p_{\perp}$ and the second adiabatic invariant, which is the integral of the particle's momentum along the guiding field line, is identically zero for particles mirroring at the magnetic equatorial plane.
Since particle energies change in the diffusion process, the radial diffusion theory deals with particle phase space densities at constant first adiabatic invariant rather than three-dimensional space densities at constant energy. The radial diffusion equation is

$$
\frac{\partial f}{\partial t}=L^{2} \frac{\partial}{\partial L}\left(\frac{D}{L^{2}} \frac{\partial f}{\partial L}\right)+\mathscr{S}(L)-\mathscr{L}(L)
$$

where $f$ is the phase space density at a particular $M, D$ is the diffusion coefficient, $L$ is the McIlwain parameter, and $\mathscr{S}(L)$ and $\mathscr{L}(L)$ represent local sources and sinks, respectively. Equation (1) can be used with measurements of the gradient of $f$ to obtain quantitative information about diffusive flows and particle losses. We therefore calculate the phase space densities of the energetic oxygen ions from the measured intensities.

The differential intensity $j$ and the integral intensity $J$ are related to the phase space density $f$ by

$$
f(p, L, \alpha)=\frac{j_{\alpha}(E, L)}{p^{2}}=-\frac{1}{p^{2}} \frac{d \dot{J}_{\alpha}(E, L)}{d E}
$$

where $E$ is the kinetic energy. Specializing to the $\alpha=90^{\circ}$ case and changing variables from $E$ and $p$ to $M$ gives the equation

$$
f\left(M, L, \alpha=90^{\circ}\right)=\frac{\gamma(M, L)}{2 m M^{2}} \frac{J_{\perp}(M, L)}{B^{2}(L)}
$$

where $\gamma=-\left[d \log \left(J_{\perp}\right) / d \log (M)\right]$. Therefore, at a given value of $M$, the change in phase space density between two radial positions $L_{1}$ and $L_{2}$ can be directly determined by measurements of the magnetic field strength, the integral intensity, and the slope of the integral intensity at $M$ :

$$
\frac{f\left(M, L_{2}\right)}{f\left(M, L_{1}\right)}=\frac{\gamma\left(M, L_{2}\right)}{\gamma\left(M, L_{1}\right)} \frac{J_{\perp}\left(M, L_{2}\right) / B^{2}\left(L_{2}\right)}{J_{\perp}\left(M, L_{1}\right) / B^{2}\left(L_{1}\right)}
$$

If spectral information is not available, it is often assumed that $\gamma\left(M, L_{1}\right)=\gamma\left(M, L_{2}\right)$. As is shown below, this assumption is not valid in the present case. The $\gamma$ 's for measurements at adjacent radial positions will typically differ by a factor of 2 , so that order of magnitude estimates of the phase space density gradient can be made by using $J_{\perp} / B^{2}$, while detailed studies require using (4).

In Figure $8, J_{\perp} / B^{2}$ is plotted as a function of $M$ at ten different radial positions in the magnetosphere. All measurements were made at or near magnetic equatorial crossings and all those except the 7.6 and $20.0 R_{J}$ points were made with LET $B$ pointing within $20^{\circ}$ of the plane perpendicular to the magnetic field direction. Magnetic equatorial crossings were identified by using $B$ field magnitude and direction data [Ness et al., 1979a, b; N. F. Ness et al., private communication,

TABLE 2. Region Definitions and Magnetic Field Values Used in Spectral Analysis

\begin{tabular}{ccccc}
\hline Region & Time & Radial Interval, $R_{\boldsymbol{J}}$ & Typical Distance, $\boldsymbol{R}_{\boldsymbol{J}}$ & Typical B Field, ${ }^{*} \mathrm{G}$ \\
\hline 1 & $64 / 1106-64 / 1235$ & $5.01-4.92$ & 5.0 & $3.3 \times 10^{-2}$ \\
$2 a$ & $64 / 0936-64 / 1106$ & $5.59-5.01$ & 5.2 & $2.8 \times 10^{-2}$ \\
$2 b$ & $64 / 1235-64 / 1405$ & $4.92-5.36$ & & \\
$3 a$ & $64 / 0842-64 / 0936$ & $6.11-5.59$ & 5.7 & $2.1 \times 10^{-2}$ \\
$3 b$ & $64 / 1405-64 / 1443$ & $5.36-5.68$ & & \\
4 & $190 / 2208-191 / 0136$ & $10.12-10.40$ & 10.2 & $3.3 \times 10^{-3}$ \\
5 & $190 / 1235-190 / 1450$ & $12.60-11.65$ & 12.1 & $1.8 \times 10^{-3}$ \\
6 & $190 / 0237-190 / 0514$ & $17.77-16.31$ & 17.2 & $6.0 \times 10^{-4}$ \\
\hline
\end{tabular}

* Magnetic field values from N. F. Ness (private communication, 1980). 


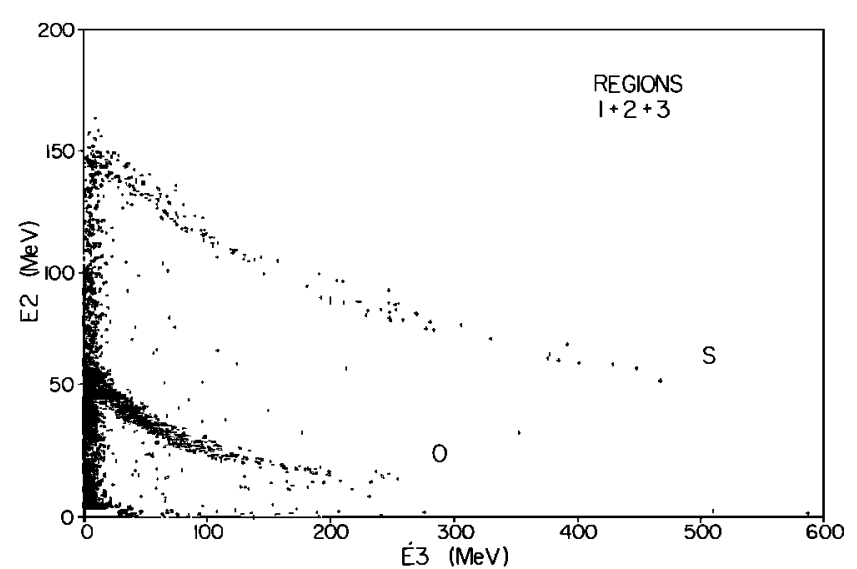

Fig. 4. Plot of energy losses $E 2$ and $E 3$ in detectors $L 2$ and $L 3$ in regions $1+2+3$ (Voyager 1 , LET B) for each pulse height analyzed event. The labelled oxygen and sulfur tracks are due to particles penetrating $L 2$ (and $L 1$ ) and stopping in $L 3$. Events in the bottom lefthand comer have not been plotted.

1980]. The 7.6 and $20.0 R_{J}$ points were obtained with LET $B$ pointing $\sim 30^{\circ}$ from the plane perpendicular to the magnetic field direction and are therefore not accurate measures of $J_{\perp}$. (If the flux varies in pitch angle as $\sin ^{4} \alpha$ as is the case for protons and electrons with $E>21 \mathrm{MeV}$ between 5 and $12 R_{\text {, }}$ [Van Allen et al., 1974], then $J_{\perp}$ at 7.6 and $20.0 R_{J}$ would be a factor $\sim 2$ higher than that plotted in Figure 8.) It is clear in Figure 8 that at a given $M, J_{\perp} / B^{2}$ increases rapidly with in-

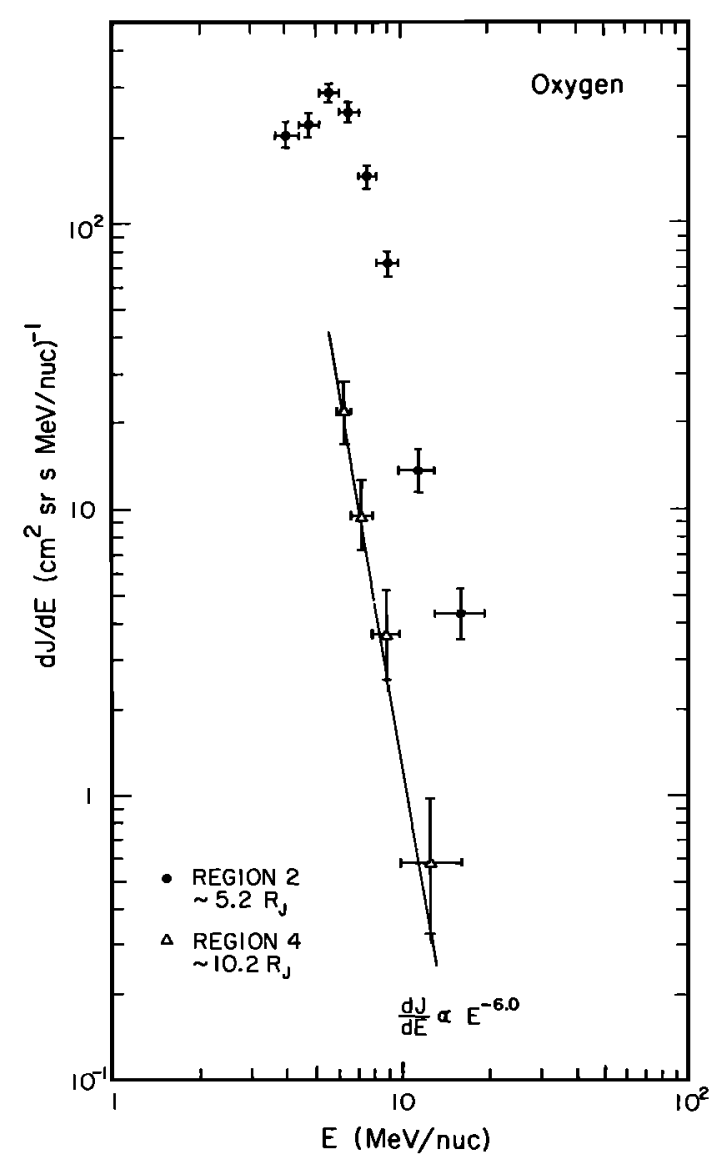

Fig. 5. Differential energy spectra of oxygen ions in regions 2 and 4. A power law of exponent 6.0 is shown for comparison with the measured data in region 4. It does not represent a fit to the data. creasing radial distance out to at least $17 R_{J}$, indicating a positive radial gradient in the phase space density in this region.

The Pioneer 10 and 11 points in Figure 8 are from the University of Chicago fission cell detector [Simpson et al., 1974, 1975] assuming that its response at $L=3.4$ (Pioneer 10) and $L$ $=1.9$ (Pioneet 11 ) was dominated by oxygen nuclei of $E>75$ $\mathrm{MeV} / \mathrm{nuc}$. Although the detector was primarily designed to measure proton fluxes in the presence of high fluxes of energetic electrons, it is also sensitive to nuclei with $Z>1$. Signal characteristics inside $10 R_{J}$ and comparison with other detector measurements at $1.9 R_{J}$ were inconsistent with a proton dominated response, leading Simpson et al. [1974, 1975] to suggest that energetic $Z>1$ nuclei were contributing to the fission cell measurements in these regions. This suggestion cannot be investigated quantitatively by using CRS data, since the observations were made at different times, but Figure 8 does indicate that it is reasonable to interpret the measurement as oxygen. If the fission cell was responding predominantly to oxygen, and if energetic oxygen fluxes were similar at all encounters, then there is little if any gradient in the phase space density at $\sim 710 \mathrm{MeV} /$ nuc-G between 5.0 and 3.4 $R_{\boldsymbol{y}}$. There is a gradient at $\sim 130 \mathrm{MeV} /$ nuc- $\mathrm{G}$ between 5.0 and $1.9 R$, which is not surprising considering the absorption effect of Amalthea ( $2.5 R_{J}$ ) on inwardly diffusing particles. A further analysis of the fission cell data in light of the Voyager findings could provide useful information about energetic oxygen fluxes in the $1.6-$ to $4.9-R_{J}$ region where no other spacecraft has been.

In order to calculate the radial dependence of the phase space density at constant magnetic moment, $J_{\perp} / B^{2}$ was determined as a function of $L$ for discrete values of $M$ from the spectra in Figure 8, and is shown for a few representative values in Figure 9. Since all measurements were made near magnetic equatorial plane crossings, the $L$ used in this paper is defined to be equal to the radial distance in $R_{J}$. (These $L$ values may differ from the actual $L$ values by up to $\sim 0.1$ due to the dipole offset.) The data in Figure 9 indicate a steep outward gradient in $J_{\perp} / B^{2}$. In addition, although no single constant $M$

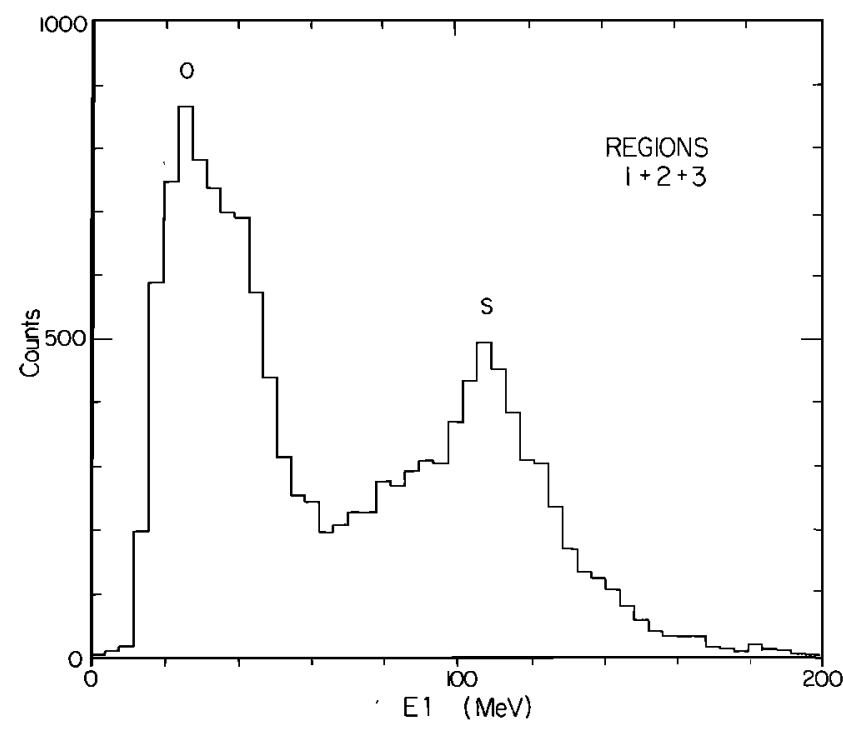

Fig. 6. Pulse-height histogram of the $L 1$ detector in regions $1+2$ +3 . The maxima correspond to oxygen and sulfur as shown. Since only those events satisfying the equation $E 1+0.4 E 2+0.2 E 3>9.6$ $\mathrm{MeV}$ have been plotted, the sharp decrease in the oxygen maximum below $\sim 20 \mathrm{MeV}$ is probably a threshold effect. 
TABLE 3. Livetime Corrections for the $Z>2$ Rate

\begin{tabular}{ccccc}
\hline Region & Measured $Z>2$ Rate, $\mathrm{s}^{-1}$ & Correction Factor** & $\begin{array}{c}\text { Effective Oxygen } Z>2 \\
\text { Threshold, } \dagger \text { MeV/nuc }\end{array}$ & $\begin{array}{c}\text { Oxygen Fraction } \\
\text { of } Z>2 \text { Rate } \ddagger\end{array}$ \\
\hline 1 & $1.2 \times 10^{4}$ & 1.3 & 1.4 & 0.52 \\
2 & $1.0 \times 10^{4}$ & 1.2 & 1.4 & 0.53 \\
3 & $1.3 \times 10^{4}$ & 1.3 & 1.4 & $\sim 0.5 \S$ \\
4 & $1.8 \times 10^{4}$ & 1.4 & 1.3 & $\sim 0.5 \S$ \\
5 & $1.4 \times 10^{4}$ & 1.2 & 1.2 & $\sim 0.5 \S$ \\
6 & $9.0 \times 10^{2}$ & 1.01 & 1.1 & $\sim 0.5 \S$ \\
\hline
\end{tabular}

Typically \pm 0.1 .

$\dagger 1.1 \mathrm{MeV} / \mathrm{nuc}$ is the nominal oxygen threshold level. Sulfur threshold is $0.2 \mathrm{MeV} / \mathrm{nuc}$ lower than oxygen threshold.

$\ddagger$ Note: not abundance fraction.

I 1.0 implies no correction.

8 Extrapolated from spectra at higher energy. Typically \pm 0.1 .

curve covers the entire radial range, all measured curves show the same trend of increasing outward gradient toward smaller values of $L$ between 17.2 and $5.7 R_{J}$. Inside $5.7 R_{J}$, however, smaller gradients are seen at smaller values of $L$. The data also show that the gradient in a given radial range is not the same for different values of $M$, but tends to be larger for larger values of $M$. This is due to the fact that the spectra in Figure 8 do not have the same spectral index (slope) at a given value of $M$. For example, the $\sim 5.7 R_{J}$ spectrum is steeper at $500 \mathrm{MeV} /$ nuc-G than the spectrum at $\sim 10.2 R_{J}$, and therefore the gradi-

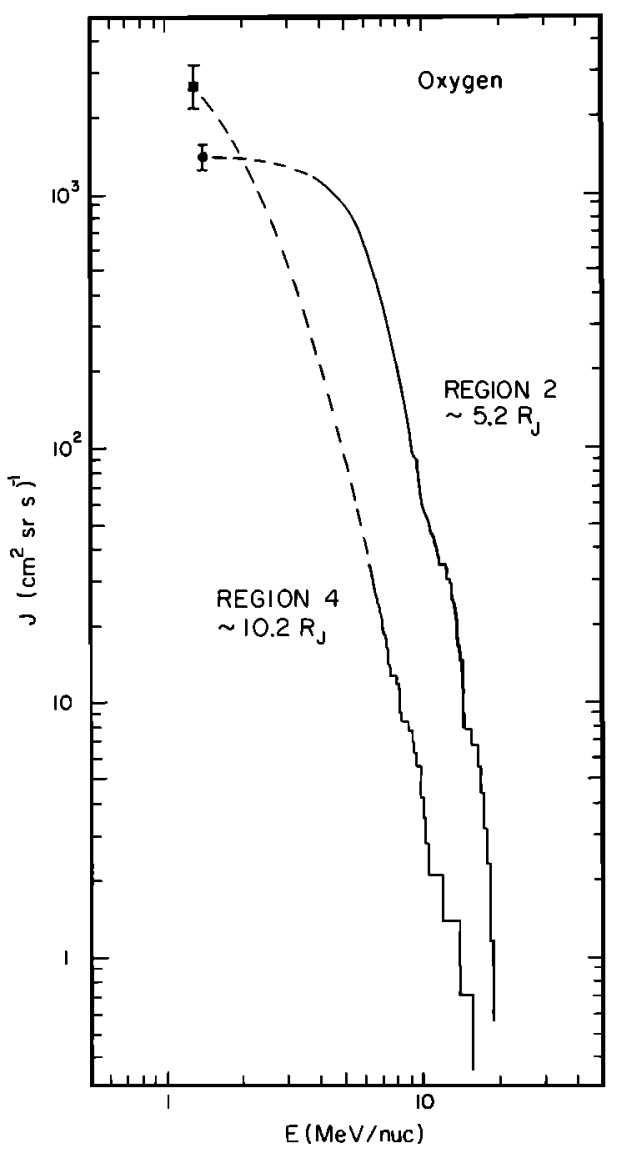

Fig. 7. Integral oxygen energy spectra in regions 2 and 4. $J(E)$ is the integral intensity above energy $E$. The solid parts of the curves represent the same data set as the differential spectra in Figure 5. No binning has been done to the data; each event is shown as a step up in integral intensity. The points at $1.4 \mathrm{MeV} /$ nuc are based on the $Z>2$ rate. ent measured between 5.7 and $10.2 R_{J}$ will increase toward larger values of $M$. Hence, in the present analysis it is not a good approximation to assume $\gamma\left(M, L_{1}\right)=\gamma\left(M, L_{2}\right)$ in (4).

Relative phase space densities at constant values of $M$ can now be calculated by using (4) and the data in Figures 8 and 9. The $f(L)$ for $M=680 \mathrm{MeV} /$ nuc-G between 5.7 and $17.2 R_{J}$ is shown in Figure 10. The points at 5.7, 10.2, and 12.1 $R_{J}$ are all based on direct measurements of $J_{\perp} / B^{2}$ and $\gamma$. These quantities were not measured at $17.2 R_{J}$ for $M=680 \mathrm{MeV} /$ nuc-G but reasonable limits can be placed on them by extrapolating the 17.2 $R_{J}$ spectrum to this $M$ value. The corresponding limits on the phase space density are shown as a bar at $L=17.2$ in the figure. The parameters used to calculate $f(L)$ are listed in Table 4. Between 5.7 and $10.2 R_{J}$ the radial dependence of the phase space density shown in Figure 10 is $f(L) \propto L^{14}$. Although there are no direct measurements in this region at this value of $M$ to confirm a power law representation, the $7.6 R_{J}$ point at $160 \mathrm{MeV} /$ nuc-G (Figure 8) and reasonable assumptions concerning the spectrum indicate that $f(L=7.6)$ at 680 $\mathrm{MeV} /$ nuc-G should be between $10^{-2}$ and $10^{-1}$. The power law representation falls within this interval at $f(7.6) \approx 2 \times 10^{-2}$.

\section{Diffusion EQuation Solutions}

The radial dependence of $f$ shown in Figure 10 is now compared with solutions of the diffusion equation (1) to obtain limits on the diffusion coefficient and the particle loss rate. It is assumed that the measured intensities represent a steady state condition and that there are no sources of energetic oxygen ions between 5.7 and 17.2 $R$. With a loss term of the form $\mathscr{L}=f / \tau$, where $\tau$ is the particle loss lifetime, equation (1) then becomes

$$
L^{2} \frac{\partial}{\partial L}\left(\frac{D}{L^{2}} \frac{\partial f}{\partial L}\right)-\frac{f}{\tau}=0
$$

A technique often used in solving this equation is to assume

$$
D=D_{0} L^{n} \quad \tau=\tau_{0} L^{m}
$$

[Haerendel, 1968; Barbosa and Coroniti, 1976; Baker and Goertz, 1976; Goertz et al., 1979; Richardson et al., 1980] where $D_{0}, \tau_{0} n$, and $m$ are constants to be determined by fitting the solution to the data. Three radial diffusion mechanisms that theoretically give a power law form for the diffusion coefficient are fluctuating electric fields due to ionospheric turbulence ( $n=3$ [Brice and McDonough, 1973]), disturbances in the convection electric field due to changes in the solar wind interaction with the magnetosphere ( $n=6$ [Birmingham, 1969]), and magnetic field disturbances due to solar wind 


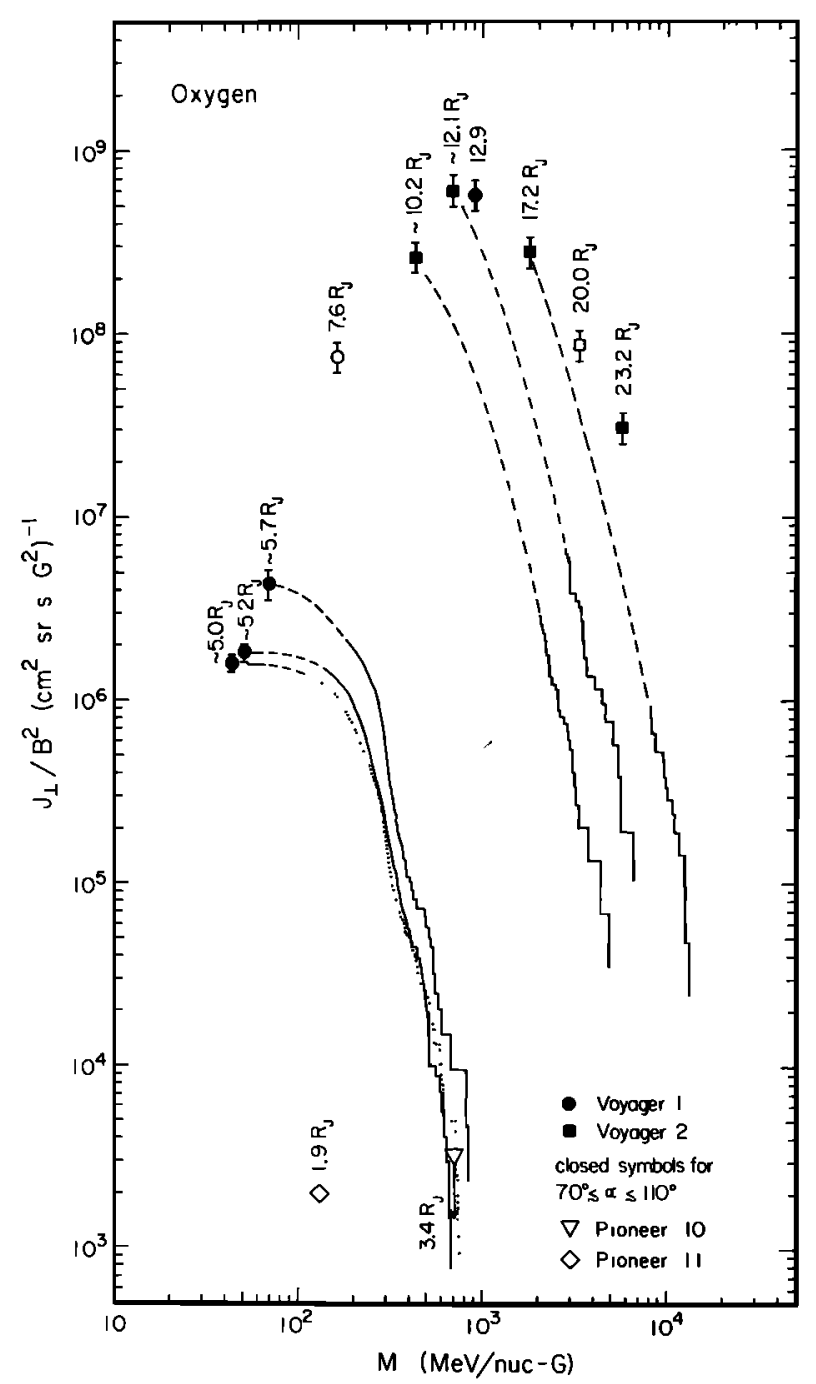

Fig. 8. Voyager 1 and 2 measurements of $J_{\perp} / B^{2}$ as a function of $M$ for oxygen at different radial positions in the magnetosphere. All measurements were made at, or near, magnetic equatorial crossings. The telescope pointing direction was within $20^{\circ}$ of the plane perpendicular to the local magnetic field direction for the closed symbols and was $\sim 30^{\circ}$ from the plane for the open symbols. (The pitch angles $\alpha$ of the measured particles are on average equal to the telescope pointing direction.) The dashed lines represent interpolations between the spectral data and the $Z>2$ rate points. The spectrum at $\sim 5.0 R$, is dotted to distinguish it from the spectrum at $\sim 5.2 R_{\mathbf{J}}$. The Pioneer 10 and 11 points are estimates of the oxygen intensity based on data from the University of Chicago fission cell detector [Simpson et al., 1974, 1975]. It was assumed that the detector was responding primarily to oxygen nuclei and that the spectral index of the oxygen spectrum was $\sim 3$ (approximately the value used by the authors). For a steeper assumed spectrum, the points could be as much as a factor of 4 lower.

pressure variations ( $n=10$ [Nakada and Mead, 1965]). Another possible diffusion mechanism is magnetic flux tube interchange driven by centrifugal plasma forces. The $L$ dependence of $D$ has not been fully specified theoretically for this process, but indications are that $D$ will not be a strict power law in $L$ [Siscoe and Summers, 1981]. Plasma observations between 6 and $8 R_{j}$, however, are consistent with the approximation of a power law $D$ with $n \approx 5-8$ [Froidevaux, 1980; Siscoe and Summers, 1981]. A power law $\tau$ cannot be justified to the same extent as a power law $D$ but is approximately obtained in the strong pitch angle diffusion limit. In this case

$$
\tau_{\text {strong }}=\frac{\tau_{b} / 4}{\alpha_{01}{ }^{2} / 2}
$$

[Kennel and Petschek, 1966] where $\tau_{b}$ is the particle bounce period and $\alpha_{01}$ is the equatorial loss cone angle. For nonrelativistic particles at constant $M$ in a dipolar magnetic field,

$$
\tau_{\text {strong }}=\frac{R_{J}}{c}\left(\frac{m_{p} c^{2}}{M B_{S}}\right)^{1 / 2} L^{11 / 2}\left(4-\frac{3}{L}\right)^{1 / 2}
$$

where $m_{p}$ is the proton mass for $[M]=\mathrm{MeV} /$ nuc-G and $B_{S}$ is the equatorial surface field of Jupiter, approximately equal to 4G. For large $L, \tau_{\text {strong }} \propto L^{11 / 2}$.

As a preliminary step, (5) can be solved for the special case of lossless diffusion $(\tau \rightarrow \infty)$. Given the two boundary conditions $f\left(L_{1}\right)$ and $f\left(L_{2}\right)$, the lossless diffusion solution for $n \neq 3$ is

$$
f(L)=\frac{f\left(L_{1}\right)\left(L^{3-n}-{L_{2}}^{3-n}\right)-f\left(L_{2}\right)\left(L^{3-n}-L_{1}{ }^{3-n}\right)}{L_{1}{ }^{3-n}-L_{2}{ }^{3-n}}
$$

The parameter $D_{0}$ does not appear in this equation because it is a multiplicative constant in the general solution that disappears when the boundary conditions are imposed. A search can now be made for values of $L_{1}, L_{2}, f\left(L_{1}\right), f\left(L_{2}\right)$, and $n$ that give acceptable fits to the measured phase space densities. An acceptable solution is defined as one that agrees with the data

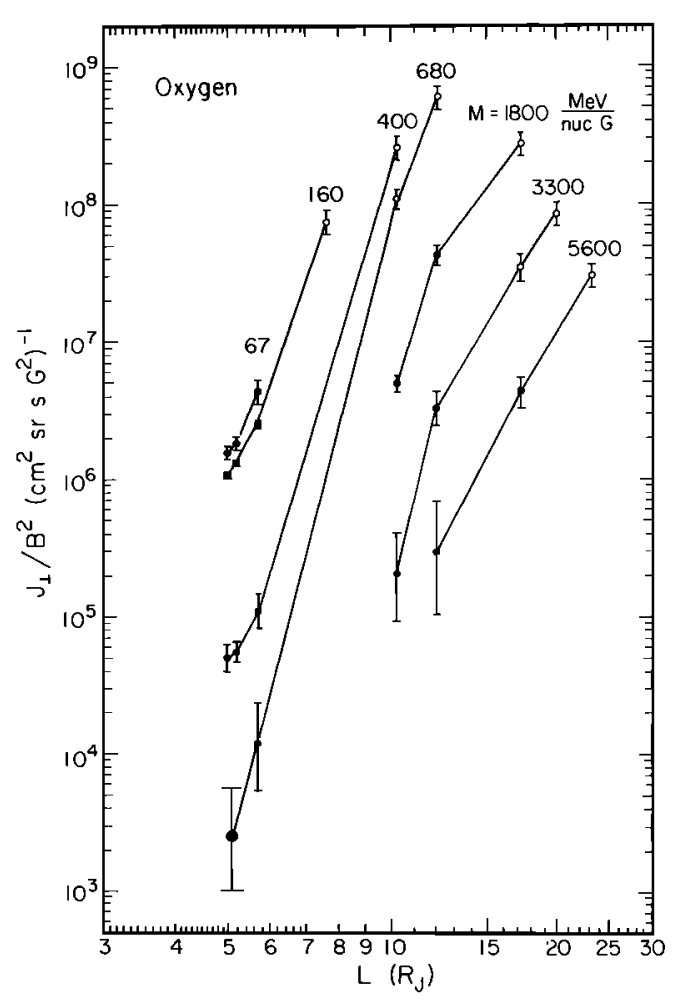

Fig. 9. Measurements of $J_{\perp} / B^{2}$ as a function of $L$ for various values of $M$. The curves represent cuts at constant $M$ in Figure 8. The open symbols signify $J_{\perp} / B^{2}$ values derived primarily from $Z>2$ rate measurements. The errors on these points are due mainly to uncertainties in the sulfur and oxygen contributions to the rate and should be considered as systematic errors. The errors on the points with closed symbols are due to statistical uncertainties associated with the number of events in the spectrum and are therefore uncorrelated. The 5.0 and $5.2 R_{J}$ measurements at $680 \mathrm{MeV} /$ nuc-G were averaged together to improve statistics. 


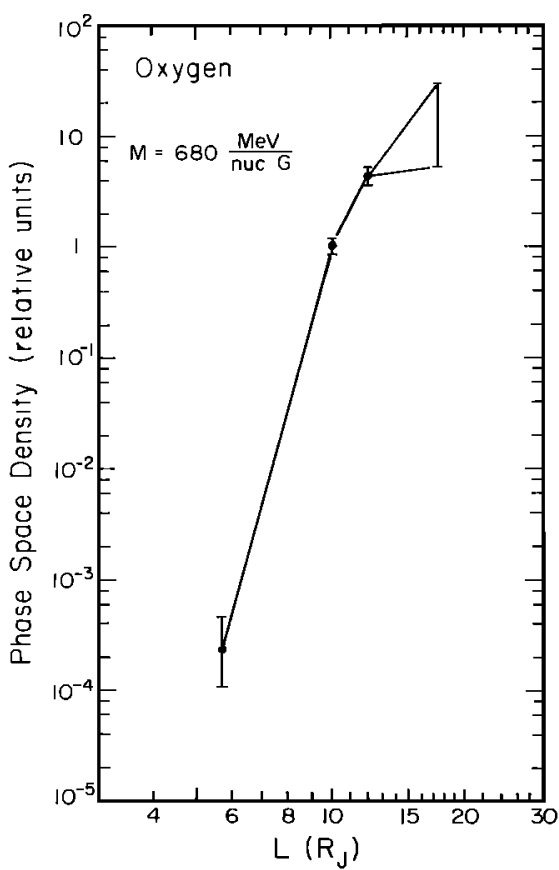

Fig. 10. The phase space density of oxygen ions with $M=680$ $\mathrm{MeV} /$ nuc-G between $L=5.7$ and $L=17.2 . f(L)$ is normalized to be 1.0 at $10.2 R_{J}$. For $L>12.1, f(L)$ was estimated by extrapolation of measurements at larger values of $M$. The uncertainty in this extrapolation is indicated by the error bar at $L=17.2$.

at 5.7, 10.2, and 12.1 $R_{J}$ and with the limits at $17.2 R_{J}$ (Figure 10) and has an essentially monotonic radial dependence (i.e., no large local maxima or minima). Figure $11 a$ shows three lossless diffusion solutions. None of these solutions, or any other ones tried, is acceptable as defined above. Solution 1 is the closest to an acceptable fit over the entire radial range, but it, as well as solution 2 , has negative values for $n$ while theory and other observations indicate that positive values are more likely. Solution 3 has $n=0$, but fits (approximately) only the measurements between $\sim 8$ and $17.2 R_{\mathrm{J}}$. Although no solution with non-negative $n$ was found to fit the data over the entire radial range, solution 3 , which is our best effort in terms of finding a solution with non-negative $n$ that fits the data to the smallest possible radial distance, shows that lossless diffusion may be occurring outside $\sim 8 R_{\boldsymbol{J}}$. Thus, there is evidence that loss processes must be occurring inside of $\sim 8 R$, and may be occurring at larger radial distances. It is not surprising that losses are occurring inside $\sim \mathbf{8} R_{J}$ since both the plasma density [Bagenal and Sullivan, 1981] and the electromagnetic wave activity [Scarf et al., 1979] are enhanced in this region. The wave enhancement continues out to $\sim 10 R_{J}$, indicating that losses are also likely in the 8-10 $R$, region.

As a preliminary investigation of the effect of loss processes on the phase space density, losses of the form given by (6) are

TABLE 4. Quantities Used to Calculate the Phase Space Density at $M=680 \mathrm{MeV} /$ nuc-G between $L=5.7$ and $L=17.2$

\begin{tabular}{rrccc}
\hline \multicolumn{1}{c}{$L_{1}$} & $L_{2}$ & $\gamma\left(M, L_{2}\right)$ & $J_{\perp}\left(M, L_{2}\right) / B^{2}\left(L_{2}\right)$ & $f\left(L_{2}\right) / f\left(L_{1}\right)$ \\
\hline & 5.7 & 4.8 & $1.2 \times 10^{4}$ & \\
5.7 & 10.2 & 2.2 & $1.1 \times 10^{8}$ & 4200. \\
10.2 & 12.1 & 1.7 & $6.1 \times 10^{8}$ & 4.3 \\
12.1 & 17.2 & & & \\
upper limit & 1.7 & $3.9 \times 10^{9}$ & 6.5 \\
lower limit & 0.8 & $1.6 \times 10^{9}$ & 1.2 \\
\hline
\end{tabular}

now included in the diffusion equation. The general solution in this case with boundary conditions $f\left(L_{1}\right)$ and $f\left(L_{2}\right)$ is

$$
\begin{aligned}
f(L) & =f\left(L_{1}\right)\left(\frac{L_{1}}{L}\right)^{(n-3) /(2)} \frac{I_{\nu}\left(x_{2}\right) K_{\nu}(x)-K_{\nu}\left(x_{2}\right) I_{\nu}(x)}{I_{\nu}\left(x_{2}\right) K_{\nu}\left(x_{1}\right)-K_{\nu}\left(x_{2}\right) I_{\nu}\left(x_{1}\right)} \\
& +f\left(L_{2}\right)\left(\frac{L_{2}}{L}\right)^{(n-3) /(2)} \frac{K_{\nu}\left(x_{1}\right) I_{\nu}(x)-I_{\nu}\left(x_{1}\right) K_{\nu}(x)}{K_{\nu}\left(x_{1}\right) I_{\nu}\left(x_{2}\right)-I_{\nu}\left(x_{1}\right) K_{\nu}\left(x_{2}\right)}
\end{aligned}
$$

[see, e.g., Goertz et al., 1979] where $I_{\nu}(x)$ and $K_{\nu}(x)$ are the modified Bessel functions of order $\nu, \nu=(3-n) /(n+m-2)$, and $x_{i}=x_{0} L_{i}^{-p}$, where $p=(n+m-2) / 2$ and $x_{0}=\left(p^{2} D_{0} \tau_{0}\right)^{-1 / 2}$. There is also a special solution for $n+m=2(p=0)$, but it does not apply here since, as will be shown below, the data specify $n+m \approx 5-6$. Equation (10) is usually simplified considerably by choosing $f(L=1)=0$ or $f(L=0)=0$. In the present analysis, however, the inner boundary condition was chosen to be the measured density at $L=5.7, f(5.7)=2.4 \times$ $10^{-4}$, because it is likely that the diffusion coefficient and lifetime are much different inside Io's orbit than outside given the abrupt change in plasma density and temperature at $L \approx 5.7$ [Bagenal and Sullivan, 1981; Richardson and Siscoe, 1981]. The other condition applied was $f(L=10.2)=1.0$.

The method used in searching for acceptable solutions for a given $m$ and $n$ was to find the $x_{0}$ such that the solution gives the correct value at $12.1 R$, and then examine the solution to see if it lies between the limits at 17.2 $R$, and has an essentially monotonic radial dependence. Of the three sample solutions shown in Figure $11 b$, only solution 2 is acceptable. Solution 1 lies outside of the range of the data at $17.2 R_{J}$, while solution 3 has a pronounced minimum at $\sim 6.5 R_{J}$. The values of $n$ and $m$ for which acceptable solutions were found are shown in Figure 12. There are no acceptable solutions for $n<3$, but for $n$ $>14$ the band of solutions continues. Although the acceptable

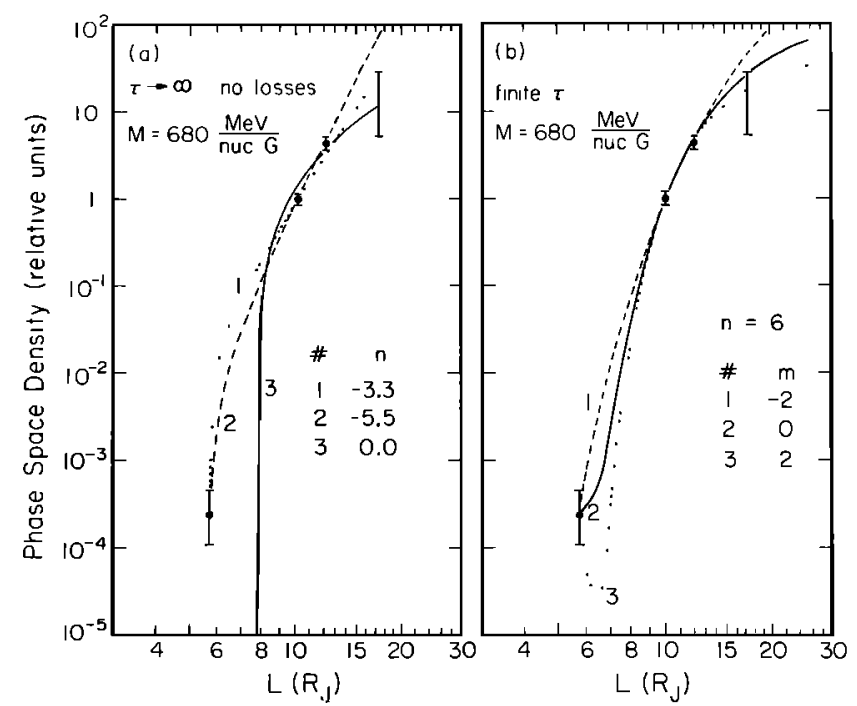

Fig. 11. Solutions of the diffusion equation compared with the measured $f(L)$ at $680 \mathrm{MeV} /$ nuc-G. (a) Lossless diffusion; equation (9). The values of $\boldsymbol{n}$ for each solution are listed. For solutions 1 and 2, $f\left(L_{2}\right)=12.0, L_{1}=8.0$, and $f\left(L_{1}\right)=3.1 \times 10^{-2}$ (i.e., the value at $8 R_{J}$ of and $L_{1}=5.7$ and $L_{2}=12.1$, respectively. For solution $3, L_{2}=17.2$, $f\left(L_{2}\right)=12.0, L_{1}=8.0$, and $f\left(L_{1}\right)=3.1 \times 10^{-2}$ (i.e., the value at $8 R_{J}$ of the power law interpolation between measurements at 5.7 and 10.2 $\left.R_{J}\right)$. No curve gives an acceptable fit to the measured values over the entire radial range. (b) Lossy diffusion; equation (10). The measured values of $f(L)$ were used for $f\left(L_{1}\right)$ and $f\left(L_{2}\right)$ with $L_{1}=5.7$ and $L_{2}=$ 10.2. Only curve 2 is an acceptable fit. 


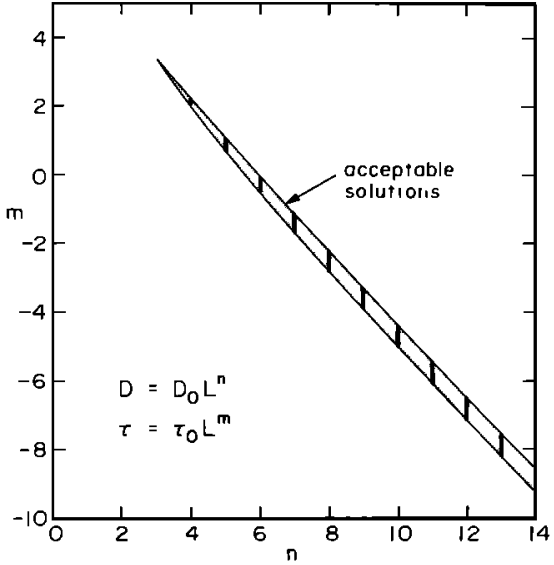

Fig. 12. The region in the $\mathrm{n}$-m plane where acceptable solutions were obtained for the case of lossy diffusion. Calculations were done only at integer $n$. The band of solutions continues for $n>14$.

$n$ and $m$ 's individually vary by about 12 units in the figure, the sum $n+m$ is relatively constant, ranging from $\sim 6$ at $n=3$ to $\sim 5$ at $n=14$. This is due to the fact that all parameters except $\nu$ depend on $n$ and $\boldsymbol{m}$ in sum. Since the solution depends only weakly on $\nu$, its $n$ dependence is a small effect. Also note that $D_{0}$ and $\tau_{0}$ enter the solution as a product. No information can be gained about either of them individually from this analysis alone. This is physically reasonable since, for a given radial dependence of $f, D$, and $\tau$, the larger $D_{0}$ becomes, the larger the net diffusive flux into a region becomes, and, therefore, the higher the loss rate (smaller $\tau_{0}$ ) must be to maintain a constant density in the region.

The acceptable values of $D_{0} \tau_{0}$ were found to range from $\sim 5$ $\times 10^{-7}$ at $n=3$ to $\sim 5 \times 10^{-6}$ at $n=14$. Given $D_{0} \tau_{0}, n$, and $m$, lower limits on $\tau$ can be imposed to determine the maximum allowable diffusion coefficient as a function of $L$. The minimum $\tau, \tau_{\min }$, dictated by the strong pitch angle diffusion limit, is given by (8). Since all acceptable solutions were found to have $m<11 / 2$, the strongest restriction that can be placed on $\tau$ between 5.7 and $17.2 R_{J}$ occurs at $17.2 R_{J}$ (i.e., if a given solution with $m<11 / 2$ has $\tau(5.7)=\tau_{\text {sirong }}(5.7)$, then $\tau(17.2)<$ $\tau_{\text {strong }}(17.2)$, which is impossible). At $17.2 R_{J},(8)$ gives $\tau_{\text {strong }}$ $(17.2)=1.7 \times 10^{6} \mathrm{~s}$, and therefore the minimum $\tau_{0}$ is $\tau_{0 \min }=$ $17.2^{-\mathrm{m}} 1.7 \times 10^{6} \mathrm{~s}$. The maximum diffusion coefficient, $D_{\max }$, is then

$$
D_{\max }(L)=\frac{D_{0} \tau_{0}}{\tau_{0 \min }} L^{n}=1.5 \times 10^{-5}\left|\frac{L}{17.2}\right|^{n} s^{-1}(5.7 \leq L \leq 17.2)
$$

assuming $n+m \approx 6$ and $D_{0} \tau_{0} \approx 10^{-6}$. For $L=6, D_{\max }$ is a steeply decreasing function of $n$, ranging from $\sim 6 \times 10^{-7} \mathrm{~s}^{-1}$ at $n=3$ to $<3 \times 10^{-8} \mathrm{~s}^{-1}$ for $n>6$. Previous proton, electron, and plasma measurements give typical values of $3 \times 10^{-8}-4$ $\times 10^{-6}$ for the radial diffusion coefficient at $6 R_{J}$ [Thomsen et al., 1977; Goertz et al., 1979; Froidevaux, 1980; Siscoe and Summers, 1981]. These other measurements suggest that the most likely solutions in the present analysis are those with $n<$ $6(m>0)$.

For the case of finite lifetime, all acceptable solutions with $n$ $<6$ give values of the phase space density at $17.2 R$, near the upper limit allowed by the data. If the actual value is near the lower limit, there are no solutions with reasonable diffusion coefficients that fit all the data from 5.7 to $17.2 R_{J}$ given the power law forms of the diffusion coefficient and lifetime in (6). However, if losses occur only inside $8 R_{J}$, the strong pitch angle diffusion lifetime at 17.2 $R$, will not be a constraint on $\tau_{\min }$ and $D_{\max }$ at $6 R_{J}$. If, for example, we assume the above lossy-diffusion solution inside $8 R$, with $n+m \approx 6, D_{0} \tau_{0} \approx$ $10^{-6}$, and $\tau_{\text {strong }}(L=8)=2.5 \times 10^{4} \mathrm{~s}$, which limits $\tau_{0 \mathrm{~min}}$ to $8^{-m}$ $2.5 \times 10^{4} \mathrm{~s}$, then the equivalent of (11) becomes

$$
D_{\max }(L)=1.1 \times 10^{-5}\left(\frac{L}{8}\right)^{n} s^{-1} \quad(5.7 \leq L \leq 8)
$$

At $L=6, D_{\max }(6)$ ranges from $4 \times 10^{-6} \mathrm{~s}^{-1}$ to $2 \times 10^{-6} \mathrm{~s}^{-1}$ as $n$ ranges from 3 to 6 .

Since both the solution with lossy diffusion between 5.7 and 17.2 $R_{J}$ and that with lossy diffusion between 5.7 and $8 R_{J}$ and lossless diffusion between 8 and $17.2 R_{J}$ give values of the diffusion coefficient that are consistent with earlier work and give reasonable fits to the data, this data set alone cannot be used to choose between them. The reason for this inadequacy is that the data set has been restricted to magnetic equatorial crossings to simplify the analysis, and therefore does not have the necessary radial resolution to further specify the extent of the lossy region. More information can be obtained about the particle losses by an analysis that includes the nonequatorial data (work in progress) and by direct comparison with plasma and electromagnetic wave data. However, the present analysis does demonstrate the inward flow of the energetic ions and does show that the measurements can be described by a diffusion model with diffusion coefficients similar to those derived by others for electrons, protons, and plasma. Therefore, a reasonable value for the diffusion coefficient can be chosen for the source strength analysis that follows.

\section{DISCUSSION}

Having established the inward diffusive flow of the energetic oxygen ions, we can now use the measured ion number densities (as determined from the spectra), the measured phase space density gradients, and the calculated diffusion coefficients to estimate the inward diffusive flow rate of energetic oxygen ions across a given radius. This can then be compared with the Io source strength of oxygen and sulfur ions.

The diffusive flow $I$ of ions with magnetic moment greater than $M$ across radius $r$ is given by

$$
I_{>M}=n_{>M} 2 \pi r \cdot 2 h v_{D} \text { ions s }{ }^{-1}
$$

where $n_{>M}$ is the number density of oxygen ions with magnetic moment greater than $M, h$ is the scale height of the particle distribution relative to the magnetic equatorial plane, and $v_{D}$ is the radial diffusion velocity defined as $v_{D}=D \cdot[(1 /$ $f)(\partial f / \partial L)]$ [Schulz and Lanzerotti, 1974]. For the specific case of $r=10 R$, and $M=400 \mathrm{MeV} / \mathrm{nuc}-\mathrm{G}$, the oxygen number density can be estimated from the $10.2 R_{J}$ spectrum to be $n_{>M}$ $\approx 8 \times 10^{-6} \mathrm{~cm}^{-3}$. We use $10 R$, rather than a larger radial distance in this calculation to allow comparison with other diffusion coefficient determinations. The diffusion coefficient upper limit at $L=10$ obtained for the case of lossy diffusion between 5.7 and $17.2 R_{J}$ (equation (11)) ranges from $6 \times 10^{-7}$ to 3 $\times 10^{-6} \mathrm{~s}^{-1}$ for $6 \geq n \geq 3$, while that obtained for lossy diffusion between 5.7 and $8 R_{J}$ (equation (12)) ranges from $2 \times$ $10^{-5}$ to $10^{-4} \mathrm{~s}^{-1}$ for $3 \leq n \leq 10$. The results of other diffusion coefficient determinations [Thomsen et al., 1977; Goertz et al., 
1979; Froidevaux, 1980; Siscoe and Summers, 1981] can be represented by $D(L=10)=10^{-5 \pm 1} \mathrm{~s}^{-1}$ (in some cases we have used a formula quoted only for $6 \leq L \leq 8$ ) which is approximately consistent with our values. With $(1 / f)(\partial f / \partial L) \approx 1.4$ (Figure 10 ) and $h \approx 1 R_{J}$, estimated from the magnetic latitude dependerice of the $Z>2$ fluxes, the energetic oxygen flow rate in across $r=10 R_{J}$ is then $I_{>M} \approx 5 \times 10^{21 \pm 1}$ ions s ${ }^{-1}$ for $M=400 \mathrm{MeV} /$ nuc-G. Thus, since the energetic ion abundances imply an Io source, and, since the Io source strength of oxygen and sulfur ions is estimated to be $10^{27}-10^{29}$ ions $s^{-1}$ [Eviatar and Siscoe, 1980; Shemansky, 1980], we find that $\sim 10^{-7 \pm 2}$ of the ions supplied by Io to the torus at $\sim 10^{-4} \mathrm{MeV} /$ nuc-G must be accelerated to $>400 \mathrm{MeV} /$ nuc- $\mathrm{G}$ in some region outside $17 R_{J}$ and then diffuse back in to $10 R_{J}$.

Oxygen ions with magnetic moments in the range $10^{2}-10^{4}$ $\mathrm{MeV} /$ nuc-G have also been observed by the low energy charged particle (LECP) instrument just inside the magnetosphere at $65 R$, inbound on Voyager 1 [Krimigis et al., 1979], and in the magnetospheric wind region ( $r>150 R$, outbound) on both Voyagers 1 and 2 [Krimigis et al., 1981]. The $J / B^{2}$ calculated from the $65 R_{J}$ measurement is two to three orders of magnitude larger, at the same value of $M$, than that at $\mathbf{1 7 . 2}$ $R_{J}$ in Figure 8. This observation does not necessarily imply particle flow directly in from 65 to $17 R$, since there may be a maximum in the phase space density at some intermediate distance, but it does require an acceleration region outside 17 $\boldsymbol{R}_{\boldsymbol{J}}$.

The observations suggest that plasma ions diffuse outward from the Io torus, are nonadiabatically accelerated in some region outside $17 R_{J}$, and then diffuse inward and outward from the acceleration region. The radial diffusion process will change particle energies but is assumed to conserve magnetic moments. The LECP measurements just described and the CRS measurements reported here both require an acceleration mechanism that is capable of increasing particle magnetic moments from that of the plasma near Io, $\sim 10^{-4} \mathrm{MeV} /$ nuc-G, up to $\sim 10^{4} \mathrm{MeV} /$ nuc-G.

One possible mechanism is that proposed by Eviatar et al. [1976] to predict the observed energetic sodium component in the magnetosphere. In that model, fast neutral particles, possibly produced by charge exchange between neutrals and corotating plasma near Io, escape the magnetosphere and become ionized in the solar wind. The ions are then accelerated to the $\sim 400 \mathrm{~km} \mathrm{~s}^{-1}$ solar wind speed and re-enter the magnetosphere. This mechanism, will, however, result in magnetic moments of not more than $\sim 20 \mathrm{MeV} / \mathrm{nuc}-\mathrm{G}$. Two other possibilities are stochastic acceleration by cyclotron waves in the plasma of the outer magnetosphere [Papadopoulos et al., 1980], or the application of recirculation models (Sentman et al. [1975] (radial recirculation) and Goertz [1978] (azimuthal recirculation)) to heavy ions.

\section{CONCLUSIONS}

The principal conclusions of this study of oxygen and sulfur ions with energies in the range 1-20 MeV/nuc between 5 and $20 R_{J}$ in the Jovian magnetosphere are as follows:

1. The Voyager 1 oxygen and sulfur fluxes inside $\sim 18 R_{J}$ were the same as those of Voyager 2 to within a factor of $\sim 2$.

2. The minima in the oxygen and sulfur fluxes near Io occurred at $L=5.6$ inbound and $L=5.4$ outbound. These radial positions are more closely correlated with changes in plasma and electromagnetic wave conditions than with the orbital position of Io itself. The indication is, therefore, that the particle losses are due to wave-particle interactions rather than geometric absorption by Io.

3. There is a steep positive radial gradient in the phase space density of the energetic oxygen ions between 6 and 17 $R_{j}$. The diffusive flow of the energetic ions is therefore inward in this region, indicating that the acceleration region is outside $17 R_{\text {s. }}$.

4. Steady-state solutions of the diffusion equation with diffusion coefficient and loss lifetime of the forms $D=D_{0} L^{n}$ and $\tau=\tau_{0} L^{m}$ were fit to the measured phase space densities at $M=680 \mathrm{MeV} / \mathrm{nuc}-\mathrm{G}$ between 5.7 and $17.2 R_{\boldsymbol{J}}$. For the case of lossless diffusion $(\tau \rightarrow \infty)$, no solutions with reasonable values of $\boldsymbol{n}$ could be found to fit the data over the entire radial range. However, solutions were found that fit outside $8 R_{\boldsymbol{J}}$. It is therefore possible that the high-energy ions are undergoing lossless diffusion from 17.2 to $\sim 8 R_{\boldsymbol{J}}$ and then lossy diffusion from 8 to $5.7 R_{J}$. Another possibility is that there are losses of the power law form given above throughout the region. For this case, solutions were found that fit the data over the entire range 5.7-17.2 $R$, with $n+m \approx 6$ and $n>3$. The upper limit on the diffusion coefficient at $L=6$, as determined by the solutions, falls in the range of other measurements $\left(3 \times 10^{-8}-4\right.$ $\times 10^{-6} \mathrm{~s}^{-1}$ ) for $n$ between 6 and 3 .

5. On the basis of the diffusion coefficient upper limits obtained from the phase space density fits, the upper limit on the number of oxygen ions with magnetic moment greater than $400 \mathrm{MeV} / \mathrm{nuc}-\mathrm{G}$ diffusing in across $10 R_{J}$ is $\sim 5 \times 10^{21 \pm 1}$ ions $\mathrm{s}^{-1}$, indicating that $\sim 10^{-7 \pm 2}$ of the ions from Io are accelerated to $>400 \mathrm{MeV} /$ nuc-G and diffuse in to $10 R_{J}$.

\section{APPENDIX}

The high radiation levels of Jupiter's inner magnetosphere cause three instrumental effects in the LET system that must be corrected for before absolute particle fluxes and energies can be determined:

1. Discriminator retrigger times. Finite retrigger times for discriminator circuits cause a dead time in the circuits that results in observed rates that are lower than the true rates. For the CRS instrument, the effect becomes significant above rates of $10^{4} \mathrm{~s}^{-1}$.

2. Discriminator threshold shifts. High counting rates cause shifts in the baseline voltage level at the output of the detector amplifiers. Since discriminator thresholds are set for a specified level above the nominal baseline, the baseline shift produces an increase in the effective threshold, so that, for instance, at high counting rates it takes a larger energy deposit to trigger a given threshold than at low counting rates. The effect becomes significant above an energy loss deposition rate in the detector of $\sim 10^{5} \mathrm{MeV} \mathrm{s}^{-1}$. For regions 1,2 , and 3, the $\mathrm{Ll}$ energy loss distribution shown in Figure 6 produces significant baseline shifts for $Z>2$ rates above $10^{3} \mathrm{~s}^{-1}$. In regions 4 , 5 , and 6, the bulk of the particles have energy losses in $L 1 \leqslant 10$ $\mathrm{MeV}$, requiring $Z>2$ rates of at least $10^{4} \mathrm{~s}^{-1}$ before baseline shifts become important.

3. Pulse pileup effects. As fluxes increase, the probablility that two particles will be coincident in a detector within the instrumental resolving time increases, degrading the pulse height data from that detector. For large oxygen pulses in the detector, the effect of a high rate of coincident small proton pulses is to broaden the oxygen pulse height distribution. Oxygen events in coincidence with other large pulses, however, will be displaced completely out of the normal oxygen distribution. The fraction displaced is $\tau_{\mathrm{PHA}} \boldsymbol{R}_{\mathrm{Z}>2}$, where $\boldsymbol{R}_{\mathrm{Z}>2}$ is the 
TABLE 5. Livetime Corrections for Event Analysis

\begin{tabular}{ccccc}
\hline Region & $\begin{array}{c}\text { Energy Range, } \\
\text { MeV/nuc }\end{array}$ & $\begin{array}{c}\text { Livetime } \\
\text { Correction Factor }\end{array}$ & $\begin{array}{c}\text { Pulse Pileup } \\
\text { Correction Factor }\end{array}$ & $\begin{array}{c}\text { Total } \\
\text { Correction Factor }\end{array}$ \\
\hline \multirow{2}{*}{1} & $3.7-7.1^{*}$ & 1.5 & 1.4 & 2.0 \\
& $7.1-23.5 \dagger$ & 1.5 & 1.0 & 1.5 \\
2 & $3.7-7.0^{*}$ & 1.5 & 1.4 & 2.0 \\
& $7.0-23.5 \dagger$ & 1.5 & 1.0 & 1.5 \\
3 & $3.7-6.3^{*}$ & 1.5 & 1.5 & 1.5 \\
4 & $6.3-23.5 \dagger$ & 1.5 & 1.0 & 1.6 \\
5 & $6.3-23.5 \dagger$ & 1.6 & 1.0 & 3.1 \\
& $4.8-5.3^{*}$ & $3.1 \neq$ & 1.0 & 4.8 \\
6 & $5.3-23.5 \dagger$ & 1.6 & 1.0 & 1.7 \\
\hline
\end{tabular}

Correction factors in the table above are typically \pm 0.1 except as noted.

* $Z$ estimate from $L 1$ versus $L 2$ plot.

$\dagger Z$ estimate from $L 2$ versus L3 plot.

$\ddagger \pm 0.5$.

counting rate of large pulses, and the effective resolving time, $\tau_{\text {PHA }}$, for pulse height analysis is $\sim 20 \mu \mathrm{s}$. Therefore, for example, in regions 1,2 , and 3 the $Z>2$ rate (large pulses) of $\sim 1.4 \times 10^{4} \mathrm{~s}^{-1}$ (Figure $2 a$; Table 3 ) will cause $\sim 30 \%$ of the oxygen pulse heights in the front detector (LI) to be displaced by up to $\sim 100 \mathrm{MeV}$.

In order to correct for the discriminator retrigger time and threshold shifts, a spare CRS instrument was calibrated by using light pulses from light emitting diodes (LEDs) situated above the detectors. As far as the detector amplifiers and electronics are concerned, the detector pulse output is the same for ionization created by the photons as that created by a charged particle, assuming the light pulse is short in comparison with the amplifier shaping time. In our case, the amplifier shaping time is approximately $2 \mu$ s and the light pulses were always shorter than 100 ns. The counting rate in the detector was controlled by the frequency of the random pulse generator driving the LED, and the pulse height was controlled by the intensity and duration of the light pulse. The effect of different particle species, energies, and fluxes incident at the same time on a detector was simulated by having several independent LEDs above it.

The standard livetime correction formula for rate $R$ is

$$
R_{\text {true }}=\frac{R_{\text {observed }}}{\left(1-\tau R_{\text {observed }}\right)}
$$

where $\tau$ is the retrigger time of the discriminators, often assumed to be a constant. The calibration showed, however, that $\tau$ is a function both of rate and pulse height distribution in the detector and ranges from $\sim 12$ to $\sim 19 \mu \mathrm{s}$ in the six regions of interest (Table 2). Therefore rate corrections were determined for each region by simulating the measured pulse height distributions and matching the observed rate. The livetime correction, $\left[1 /\left(1-\tau R_{\text {observed }}\right)\right]$, and the effective threshold for the $Z>2$ rate, as determined by the calibration, are listed for the six regions in Table 3.

Livetime corrections were also included in the energy spectra calculations. Since only a small fraction of the valid two-detector $(L 1 \cdot L 2)$ and three-detector $(L 1 \cdot L 2 \cdot L 3)$ events are pulse height analyzed, a coincidence rate is used to convert the number of analyzed events of a given elemental species and energy into a flux measurement. The livetime correction formula for the $L 1 \cdot L 2 \cdot L 3$ coincidence rate $C$ is

$$
C_{\text {true }}=\frac{C_{\text {observed }}}{\left(1-\tau_{1} R_{L 1}\right)\left(1-\tau_{2} R_{L 2}\right)\left(1-\tau_{3} R_{L 3}\right)}
$$

where $R_{L i}$ is the singles rate in detector $L i, \tau_{1}$ is the $L 1$ retrigger time, and $\tau_{2}$ and $\tau_{3}$ are the discriminator deadtimes of $L 2$ and $L 3$, respectively, Here, discriminator deadtime refers to the time after the discriminator has returned to its zero state before it can be retriggered. $\tau_{2}$ and $\tau_{3}$ are defined differently than $\tau_{1}$ because the LET coincidence logic is a strobed system triggered by $L 1$. Equation (15) is exact only in high flux environments where all detector singles counting rates are much larger than the observed coincidence rate. The calibration showed that there is almost no discriminator deadtime in $L 2$ and $L 3$ for a heavy ion following a proton event and therefore, that the $L 2$ and $L 3$ terms in equation (15) are negligible. (There is a discriminator deadtime on the order of tens of microseconds for a heavy ion following another heavy ion, but the heavy rate in $L 2$ and $L 3$ is never greater than $\sim 10^{3} \mathrm{~s}^{-1}$ ). As in the case of the $Z>2$ rate, $\tau_{1}$ was found to depend on rate and pulse-height distribution and ranges from $\sim 13$ to $\sim 15 \mu \mathrm{s}$ in the six regions of interest. The measured livetime correction factors for the coincidence rate in these regions are listed in Table 5. The first line of each region is for $L 1 \cdot L 2$ events and the second line is for $L 1 \cdot L 2 \cdot L 3$ events. Only particles stopping in $L 3$ were used in region 4 . In regions 1,2 , and 3 , the instrument was in its $L 1 \cdot L 2$ command state, which means that a particle is required to trigger $L 1$ and $L 2$ in order to be counted in the coincidence rate; no requirement is made on $L 3$. In regions 5 and 6 the instrument was configured in a $L 1 \cdot L 2 \cdot L 3$ command state to maximize background rejection. In this state, a coincident pulse is also required in $L 3$. In order for a $Z>2$ particle stopping in $L 2$ to be analyzed in this command state, there must be a pulse in $L 3$ due to, say, an accidentally coincident proton. There is therefore an additional livetime correction factor for particles stopping in $L 2$ in regions 5 and 6, which depends on the $L 3$ counting rate.

All particles stopping in $L 3$ were analyzed by using their $L 2$ and $L 3$ pulse height information. The maximum counting rate, of large oxygen and sulfur pulses in these two detectors is on the order of $10^{3} \mathrm{~s}^{-1}$ ( $L 2$ in regions 1, 2, and 3), so that pulse pileup affects $<2 \%$ of the $Z>2$ particles stopping in $L 3$. For particles stopping in $L 2$, however, the $L 1$ pulse height is required for elemental identification. The counting rate of large pulses in $L 1$ is the $Z>2$ rate, which is on the order of $10^{4} \mathrm{~s}^{-1}$ in regions $1,2,3$, and 5 (particles stopping in $L 2$ were not used 
in region 4). The resulting amount of pulse pileup can be determined from the data by comparing a spectrum for particles stopping in $L 3$ using $L 1$ in the elemental identification process with one in which only $L 2$ and $L 3$ were used. The ratio between the resulting spectra is fairly energy independent over this energy range and indicates what fraction of the events in $L 1$ are displaced out of the oxygen distribution. The ratio was therefore used as a correction factor for the $L 1 \cdot L 2$ part of the spectrum. The pulse pileup correction factor is listed in Table 5. It was found that no correction factors were required in regions 5 and 6 , even though the $Z>2$ rate in region 5 was $\sim 1.7$ $\times 10^{4} \mathrm{~s}^{-1}$. The reason is that the $Z>2$ rate in this region was dominated by $\sim 10 \mathrm{MeV}$ pulses which do not seriously contaminate oxygen signals.

Acknowledgments. We greatly appreciate the efforts of R. E. Vogt, both in his capacity as CRS Principal Investigator and as a colleague who has provided useful discussions. We are also grateful to the Caltech and Goddard groups who have supported this investigation, with special thanks to W. E. Althouse, M. F. Beazley, R. Burrell, W. R. Cook, and D. E. Stilwell. We also thank D. L. Chenette, A. C. Cummings, and T. L. Garrard for helpful discussions. N. Ness and his colleagues on the Voyager magnetometer team provided the magnetic field data used in this analysis, which we gratefully acknowledge. We are also grateful to A. W. Schardt for providing a special formatting of the magnetic field data and for useful discussions. We thank A. Eviatar for sharing with us new ideas about acceleration processes. Supported by NASA under NAS7-100, NGR 05-002-160, and NAGW-200.

The Editor thanks T. G. Northrop and R. B. McKibben for their assistance in evaluating this paper.

\section{REFERENCES}

Bagenal, F., and J. D. Sullivan, Direct plasma measurements in the Io torus and inner magnetosphere of Jupiter, J. Geophys. Res., 86, 8447, 1981.

Baker, D. N., and C. K. Goertz, Radial diffusion in Jupiter's magnetosphere, J. Geophys. Res., 81, 5215, 1976.

Barbosa, D. D., and F. V. Coroniti, Lossy radial diffusion of relativistic Jovian electrons, J. Geophys. Res., 81, 4553, 1976.

Birmingham, T. J., Convection electric fields and the diffusion of trapped magnetospheric radiation, J. Geophys. Res., 74, $2169,1969$.

Brice, N., and T. R. McDonough, Jupiter's radiation belts, Icarus, 18, 206, 1973.

Broadfoot, A. L., M. J. S. Belton, P. Z. Takacs, B. R. Sandel, D. E. Shemansky, J. B. Holberg, J. M. Ajello, S. K. Atreya, T. M. Donahue, H. W. Moos, J. L. Bertaux, J. E. Blamont D. F. Strobel, J. C. McConnell, A. Dalgarno, R. Goody, and M. B. McElroy, Extreme ultraviolet observations from Voyager 1 encounter with Jupiter, Science, 204, 979, 1979

Brown, R. A., Optical line emission from lo, Symp. Int. Astron. Union, $65,527,1974$.

Brown, R. A., and Y. L. Yung, lo, its atmosphere and optical emissions, in Jupiter, edited by T. Gehrels, University of Arizona Press, Tucson, 1976.

Eviatar, A., and G. L. Siscoe, Limit on rotational energy available to excite Jovian aurora, Geophys. Res. Lett., 7, 1085, 1980.

Eviatar, A., Yu Mekler and, F. V. Coroniti, Jovian sodium plasma, Astrophys. J. , 205, 622, 1976.

Froidevaux, L., Radial diffusion in Io's torus: Some implications from Voyager 1, Geophys. Res. Lett., 7, 33, 1980.

Goertz, C. K., Energization of charged particles in Jupiter's outer magnetosphere, J. Geophys. Res., 83, 3145, 1978.

Goert, C. K., J. A. Van Allen, and M. F. Thomsen, Further observational support for the lossy radial diffusion model of the inner Jovian magnetosphere, J. Geophys. Res., 84, 87, 1979.

Haerendel, G., Diffusion theory of trapped particles and the observed proton distribution, in Earth's Particles and Fields, edited by B. M. McCormac, Reinhold, New York, 1968.

Hamilton, D. C., G. Gloeckler, S. M. Krimigis, and L. J. Lanzerotti, Composition of nonthermal ions in the Jovian magnetosphere, $J$. Geophys. Res., 86, 8301, 1981.
Johnson, T. V., A. F. Cook II, C. Sagan, and L. A. Soderblom, Volcanic resurfacing rates and implications for volatiles on lo, Nature, 280, 746, 1979.

Kennel, C. F., and H. E. Petschek, Limit on stably trapped particle fluxes, J. Geophys. Res., 71, 1, 1966.

Krimigis, S. M., T. P. Armstrong W. I. Axford, C. O. Bostrom, C. Y. Fan, G. Gloeckler, L. J. Lanzerotti, E. P. Keath, R. D. Zwickl, J. F. Carbary, and D. C. Hamilton, Low-energy charged particle environment at Jupiter: A first look, Science, 204, 998, 1979.

Krimigis, S. M., J. F. Carbary, E. P. Keath, C. O. Bostrom, W. I. Axford, G. Gloeckler, L. J. Lanzerotti, and T. P. Armstrong, Characteristics of hot plasma in the Jovian magnetosphere: Results from the Voyager spacecraft, J. Geophys. Res., 86, 8227, 1981.

Kumar, S., The stability of an $\mathrm{SO}_{2}$ atmosphere on Io, Nature, 280, 758, 1979.

Nakada, M. P., and G. D. Mead, Diffusion of protons in the outer radiation belt, J. Geophys. Res., 70, 4777, 1965.

Ness, N. F., M. H. Acuña, R. P. Lepping, L. F. Burlaga, K. W. Behannon, and F. M. Neubauer, Magnetic field studies at Jupiter by Voyager 1: Preliminary results, Science, 204, 982, 1979a.

Ness, N. F., M. H. Acuña, R. P. Lepping, L. F. Burlaga, K. W. Behannon, and F. M. Neubauer, Magnetic field studies at Jupiter by Voyager 2: Preliminary results, Science, 206, 966, $1979 \mathrm{~b}$.

Papadopoulos, K., J. D. Gaffey, Jr., and P. J. Palmadesso, Stochastic acceleration of large $M / Q$ ions by hydrogen cyclotron waves in the magnetosphere, Geophys. Res. Lett., 7, 1014, 1980.

Pearl, J., R. Hanel, V. Kunde, W. Maguire, K. Fox, S. Gupta, C. Ponnamperuma, and $\mathrm{F}$. Raulin, Identification of gaseous $\mathrm{SO}_{2}$ and new upper limits for other gases on Io, Nature, 280, 755, 1979.

Richardson, J. D., and G. L. Siscoe, Factors governing the ratio of inward-to-outward diffusing flux of satellite ions, J. Geophys. Res., 86, $8485,1981$.

Richardson, J. D., G. L. Siscoe, F. Bagenal, and J. D. Sullivan, Time dependent plasma injection by Io, Geophys. Res. Lett., 7, 37, 1980.

Sandel, B. R., D. E. Shemansky, A. L. Broadfoot, J. L. Bertaux, J. E. Blamont, M. J. S. Belton, J. M. Ajello, J. B. Holberg, S. K. Atreya, T. M. Donahue, H. W. Moos, D. F.Strobel, J. C. McConnell, A. Dalgarno, R. Goody, M. B. McElroy, and P. Z. Takacs, Extreme ultraviolet observations from Voyager 2 encounter with Jupiter, Science, 206, 962, 1979.

Scarf, F. L., D. A. Gurnett, and W. S. Kurth, Jupiter plasma wave observations: An initial Voyager 1 overview, Science, 204, 991, 1979.

Schardt, A. W., F. B. McDonald, and J. H. Trainor, Energetic particles in the pre-dawn magnetotail of Jupiter, J. Geophys. Res., 86, 8413, 1981.

Schulz, M., and L. J. Lanzerotti, Particle Diffusion in the Radiation Belts, Springer, New York, 1974.

Sentman, D. D., J. A. Van Allen, and C. K. Goertz, Recirculation of energetic particles in Jupiter's magnetosphere, Geophys. Res. Lett., 2, 465, 1975.

Shemansky, D. E., Mass-loading and the diffusion-loss rates of the Io plasma torus, Astrophys. J., 242, 1266, 1980.

Simpson, J. A., D. C. Hamilton, R. B. McKibben, A. Mogro-Campero, K. R. Pyle, and A. J. Tuzzolino, The protons and electrons trapped in the Jovian dipole magnetic field region and their interaction with Io, J. Geophys. Res., 79, 3522, 1974.

Simpson, J. A., D. C. Hamilton, G. A. Lentz, R. B. McKibben, M. Perkins, K. R. Pyle, A. J. Tuzzolino, and J. J. O'Gallagher, Jupiter revisited: First results from the University of Chicago charged particle experiment on Pioneer 11, Science, 188, 455, 1975.

Siscoe, G. L., and D. Summers, Centrifugally driven diffusion of Iogenic plasma, J. Geophys. Res., 86, 8471, 1981.

Smith, B. A., E. M. Shoemaker, S. W. Kieffer, and A. F. Cook II, The role of $\mathrm{SO}_{2}$ in volcanism on Io, Nature, 280, 738, 1979.

Stilwell, D. E., W. D. Davis, R. M. Joyce, F. B. McDonald, J. H. Trainor, W. E. Althouse, A. C. Cummings, T. L. Garrard, E. C. Stone, and R. E. Vogt, The Voyager cosmic ray experiment, IEEE Trans. Nucl. Sci. NS-26, 513, 1979.

Stone, E. C., R. E. Vogt, F. B. McDonald, B. J. Teegarden, J. H. Trainor, J. R. Jokipii, and W. R. Webber, Cosmic ray investigation for the Voyager missions: Energetic particle studies in the outer heliosphere and beyond, Space Sci. Rev., 21, 355, 1977.

Thomsen, M. F., C. K. Goertz, and J. A. Van Allen, A determination of the $L$ dependence of the radial diffusion coefficient for protons in Jupiter's inner magnetosphere, J. Geophys. Res., 82, 3655, 1977.

Trainor, J. H., F. B. McDonald, B. J. Teegarden, W. R. Webber, and 
E. C. Roelof, Energetic particles in the Jovian magnetosphere, $J$. Geophys. Res., 79, 3600, 1974.

Van Allen, J. A., D. N. Baker, B. A. Randall, and D. D. Sentman, The magnetosphere of Jupiter as observed with Pioneer 10, 1, Instrument and principal findings, J. Geophys. Res., 79, 3559, 1974.

Vogt, R. E., W. R. Cook, A. C. Cummings, T. L. Garrard, N. Gehrels, E. C. Stone, J. H. Trainor, A. W. Schardt, T. Conlon, N. Lal, and F. B. McDonald, Voyager 1: Energetic ions and electrons in the Jovian magnetosphere, Science, 204, 1003, 1979a.
Vogt, R. E., A. C. Cummings, T. L. Garrard, N. Gehrels, E. C. Stone, J. H. Trainor, A. W. Schardt, T. F. Conlon, and F. B. McDonald, Voyager 2: Energetic ions and electrons in the Jovian magnetosphere, Science, 206, 984, $1979 b$.

\author{
Received March 11, 1981; \\ revised June 2, 1981; \\ accepted June 2,1981 .)
}

